\title{
Artificial Neural Network based Electrocardiogram Classification for Biometric Authentication
}

\author{
P. S. Gawande \\ Deptt.of Electronics Engg. Rajarshi Shahu College \\ of Engineering, Buldana,443001,MS, India
}

\author{
S. A. Ladhake \\ SIPNA College of Engineering and Technology \\ Amravati, MS, India
}

\begin{abstract}
In this study, Multilayer Perceptron (MLP) neural network model is used for the classification of electrocardiogram (ECG) signals. Out of twelve leads recorded lead II, single lead, is used for analysis. ECG samples from eight normal individuals are recorded regularly almost every month for thirty six months. Input to the network is feature vector matrix of ten features and seven statistical and three morphological features are extracted. MLP networks with single hidden layer are trained with three runs and one thousand epochs. The testing results demonstrate that the neural network is effective tool for this application and the accuracy of 99.76 is observed during experimentation.
\end{abstract}

\section{Keywords}

ECG, Biometrics, Multilayer Perceptron, Neurosolutions

\section{INTRODUCTION}

An important factor which helps in the success of classification is the choice of appropriate machine learning technique. Now a day's security is a major concern. In security systems biometrics can be used to increase the security level. Biometrics is identification of an individual based on the physiological and/or behavioral characteristics [2]. It is highly difficult to do falsification in case of biometric systems. Biometrics has a very old history. In biometrics different physiological parameters such as fingerprint, palm veins, face recognition, DNA, palm print, hand geometry, iris, retina etc and behavioral parameters such as typing rhythm, gait, signature etc. are used as a biometric treats.

ECG contains wealth of information and it is popularly used as a diagnostic tool by the physicians. The composition mechanism and electrical activity of the human heart inherit uniqueness from the individuality of DNA [3]. Thus along with all these biometric tools, electrocardiogram can also be used as tool to distinguish the individuals. ECG as a biometrics is first time used by Lena Biel [1]. The advantage of using ECG as a biometric is that ECG checks the aliveness of a person.

Various machine learning methods have been applied to improve the classification accuracy of ECG [2]. The ECG signal varies from person to person due to the differences in position, size, and anatomy of the heart, age, sex, relative body weight, chest configuration and various other factors. However, other than the changes in the rhythm, the morphology of the ECG is generally unaltered [4]. Biometric gives less accuracy as compared to traditional recognition system but it can be preferred where it is tolerable to have false rejection than the false acceptance. The benefit of using ECG as a biometric is its security level.

Prior to this, artificial neural network is used for the classification cardiac arrhythmia, for multi class ECG signal [13].

\section{RELATED WORK}

Many researchers have used electrocardiogram as a biometric for identification or verification of an individual.

Lena Biel et al. showed the possibility to use ECG as a biometric. During the experiment Lena used database of 20 subjects. A set of temporal and amplitude features are extracted. Correlation matrix is used for the analysis and accuracy of 100 percent is achieved. The data used for experimentation was of the duration of six weeks [1].

T. W. Shen et al. used ECG as a biometric by using one lead only. The technique involved in the investigation is template matching and decision based neural network. Data used for research is taken from MIT/BIH database. They later performed more extensive testing by using a larger database, containing 168 individuals and 17 temporal and amplitude features for identification. Correlation coefficient provides a measure of the similarity of the morphology of two samples of the same subject, but the research is not carried out on the similarities of the samples of different subjects. The duration of the database used is not mentioned [6].

Steven A. Israel et al. used total number of 15 features of ECG signal, of which time duration is detected between fiducial points. Linear Discriminent analysis is used for classification. The database of 29 individuals is collected during twelve repeat sessions [7].

Yongbo Wan et al. used neural network based approach to identify human subject with ECG signal. Yongbo used ECG data of the duration of only 5-30 days [9].

Yongjin Wang et al. developed system based on temporal and amplitude distance between detected fiducial points for the same Yongjin used autocorrelation and discrete cosine transform. The database is used from the MIT-BIH and PTB websites. The testing was done on the database of 13 subjects., half of the record was used as a template and remaining half of the same recording was used as a test data [10], however data was found insufficient to check the authenticity of ECG as a biometrics.

Fahim Sufi and Chan Andrian D. C. have used three measures to determine the identification of an unknown signal: Percent Residual Difference, Correlation Coefficient, and Wavelet Distance Measurement. Both of them have given different weightages. $25 \%$ weight is given to Percentage Root Mean Square Deviation, $25 \%$ weight given to correlation, and $50 \%$ is given to wavelet distance measurement. The system is tested using the data from MIT-BIH database of 15 subjects [3].

P. S. Gawande et. al., have used ECG as a biometrics even for longer duration of time with wavelet distance measurement[11]. S. M. Jadhav et. al. proposed ANN models trained by backpropagation algorithm with momentum 
learning rule to classify fifteen different ECG data with normal and cardiac arithmia and have received accuracy of $93.1 \%[12]$.

From the above literature survey, it is clear that ECG can be used for person identification. The data used by the earlier researchers is of the duration of six weeks to maximum period of four months. Many researchers have used the public data available at the websites like MIT BIH, PTB, UCI machine learning repository datasets, most of the data available at these websites is diseased and is useful for the physiological examinations or to study the history. Considering the Heart Rate Variability or Inter Pulse Interval which is beat to beat alteration in heart rate. Many authors [1, 3] have mentioned itas a future work to check the time variant nature of ECG waveform. Thus it becomes very essential to observe the effect on ECG over a long duration of time. This provides the scope to extend the work to check the authenticity to use ECG as a biometric for longer duration of time. In this work, the data used is collected from normal persons and within the duration of thirty six months. After using different neural network models for the classification of ECG signals, it is found that, MLP gives best results for signal classification.

\section{DATA DESCRIPTION}

Input to the MLP neural network model is an ECG signal of eight normal persons. Signals are noted during thirty six months. Sixty six signals of each person are taken as input, thus total 528 signals forms the database. The ECG signal is recorded using 12 lead ECG recorders, "SAMVED" manufactured by Schiller India. The sampling rate is $400 \mathrm{~s} / \mathrm{s}$. ECG with recording of one minute is made during each sample. Out of the 12 lead signals received, lead II signal is used for the analysis purpose. The length of each signal is taken in such a way that each of the signals contains at least five ECG cycles. Seven statistical features have been extracted after decomposing the ECG signal by using wavelet transform. Three morphological feature using Pan Tompkins algorithm are calculated. Fig 1 shows the feature vector matrix which is an input to the neural network.

Neural networks are built from a large number of very simple processing elements (PE). Neural network has several layers and each layer may have different number of PEs.

These PEs simply multiplies an input by a set of weights, and nonlinearly transforms the result into an output value.

Fig. 1 shows simple MLP NN. The circles are the PEs arranged in layers. The left column is the input layer, the middle column is the hidden layer, and the right column is the output layer. The lines represent weighted connections i.e., a scaling factor between PEs.

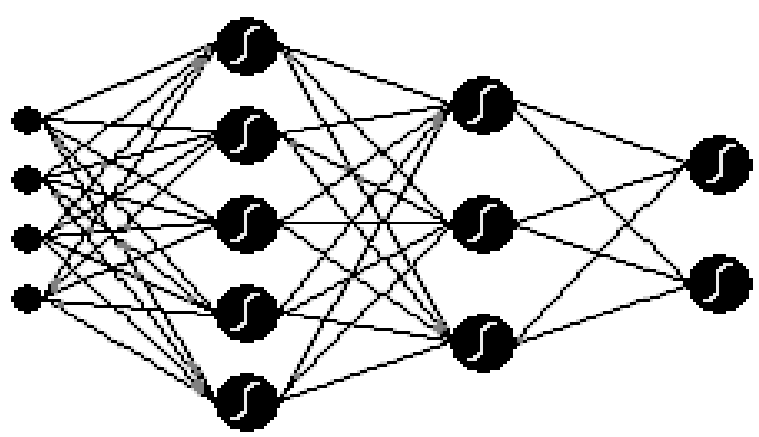

Multilayer perceptrons (MLPs) are the most common supervised neural network powerful for data modeling. With the supervised learning the network is able to learn from input and the error. MLP uses back propagation algorithm, the error generated at the output of the network is propagated back through the network to train the weights.

$\operatorname{ej}(n)=\operatorname{dj}(n)-y j(n)$

Weights in the network are calculated using gradient descent learning. Each weight in the network is fixed correcting the present value of the weight with a term that is proportional to the present input and error at the weight:

$w_{j i}(n+1)=w_{j i}(n)+\eta \delta_{i}(n) x_{j}(n)$

The local error $\delta(n)$ can be directly computed from e (n) at the output PE or can be computed as a weighted sum of errors at the internal PEs. The constant $\eta$ is called the step size. This procedure is called the backpropagation algorithm.

MLP Network is trained by using five different transfer functions and five learning rules are used for each transfer function. The percentage of data used for training, cross validation, and testing is $80: 10: 10$.

\section{PERFORMANCE MEASURE}

Seven performance measures for classification during experimentation are used such as mean squared error (MSE), normalized mean squared error (NMSE), correlation coefficient (r), Specificity, Sensitivity, Precision and Overall accuracy.

\subsection{Mean Squared Error (MSE)}

The mean squared error is simply two times the average cost. The formula for the mean squared error is:

MSE $=\frac{\sum_{j=0}^{P} \sum_{i=0}^{N}\left(d_{i j}-y_{i j}\right)^{2}}{N P}$

Where

$\mathrm{P}=$ Number of output processing elements

$\mathrm{N}=$ Number of exemplars in the data

$y_{i j}=$ network output for exemplar $i$ at processing element $j$

$\mathrm{dij}=\mathrm{desired}$ output for exemplar $\mathrm{i}$ at processing element $\mathrm{j}$

\subsection{Normalised Mean Squared Error (NMSE)}

The normalized mean squared error is defined by the following formula:

$$
\text { NMSE }=\frac{P N M S E}{\sum_{j=0}^{P} \frac{N \sum_{i=0}^{N} d_{i j}^{2}-\left(\sum_{i=0}^{N} d_{i j}\right)^{2}}{N}}
$$

Where $\mathrm{P}=$ Number of output processing elements

$\mathrm{N}=$ Number of exemplars in the data set

MSE=Mean squared error

Dij= Desired output for exemplar I at processing element $\mathrm{j}$

\subsection{Correlation Coefficient ( $r$ )}

The correlation coefficient between a network output y and and a desired output $d$ is:.

Fig. 1: Simple Multilayer Perceptron NN 
$\mathbf{r}=\frac{\frac{\sum_{\mathrm{i}}\left(\mathrm{y}_{\mathrm{i}}-\overline{\mathrm{y}}\right)\left(\left(\mathbf{d}_{\mathrm{i}}-\overline{\mathrm{d}}\right)\right)}{\mathrm{N}}}{\sqrt{\frac{\sum_{\mathrm{i}}\left(\mathrm{d}_{\mathrm{i}}-\mathrm{d}\right)^{2}}{\mathrm{~N}}} \sqrt{\frac{\sum_{\mathrm{i}}\left(\left(\mathrm{y}_{\mathrm{i}}-\overline{\mathrm{y}}\right)\right)^{2}}{\mathrm{~N}}}}$

where

$\mathrm{y}=$ network output

$d=$ desired output

During this experimentation we have considered Multilayer Perceptron (MLP) as an artificial neural network model to classify eight different classes.

\subsection{Sensitivity $(S)$}

It is the ability of the classifier to detect the positive samples as positive. It is defined as the ratio of True Positive values to the sum of True Positive and False Negative.

$$
\mathrm{S}=\frac{T_{P}}{\left(T_{P}+F_{N}\right)}
$$

\subsection{Specificity $(R)$}

It is the ability of the classifier to detect negative samples as negative. It is the ratio of True Negative values to the sum of True Negative and False Positive.

$\mathbf{R}=\frac{\mathbf{T}_{\mathrm{N}}}{\left(\mathbf{T}_{\mathbf{N}}+\mathbf{F}_{\mathrm{P}}\right)}$

\subsection{Precision}

Precision is a ratio of true positive value to sum of true positive to false negative.

$$
\text { Pracision }=\frac{T_{P}}{T_{P}+F_{N}}
$$

\subsection{Overall Accuracy}

Overall accuracy is a ratio of sum of true positive and true negative to the sum of true positive, true negative, false positive and false negative.

$$
\text { Overall Accuracy }=\frac{T_{P}+T_{N}}{T_{P}+T_{N}+F_{P}+F_{N}}
$$

True Positive ( $\boldsymbol{T}_{\boldsymbol{P}}$ ): It is the number of positive class samples detected by the classifier as positive.

True Negative ( $\left.\boldsymbol{T}_{\boldsymbol{N}}\right)$ : It is the number of negative class samples detected by the classifier as negative.

False Positive ( $\left.\boldsymbol{F}_{\boldsymbol{P}}\right)$ : It is the number of negative class samples detected by the classifier as positive.

False Negative $\left(\boldsymbol{F}_{\boldsymbol{N}}\right)$ : It is the number of positive class samples detected by the classifier as negative.

\section{RESULT}

Following are the results of classification by using MLP as a neural network model. Neurosolutions for Excel software simulation tool (Version 5.07) has been used. During classification five transfer functions (TF) are used. Transfer functions used are Tanh, Sigmoid, LinearTanh, LinearSigmoid, SoftMax and Bias, these are shown as A, B, $\mathrm{C}, \mathrm{D}, \mathrm{E}$, and F respectively in the tables 1 to 7 .

One-hidden layer $M L P N N$ is trained for three runs with 1000 epochs. The number of hidden layer $P E$ was varied from 1 to 30 with step of 1 for all transfer functions.

For each of this transfer function learning rule used are Momentum, Conjugate Gradient, Levenberg-Marquardt, Quickprop, and Delta Bar Delta and are shown as G, H, I, J and $\mathrm{K}$ respectively in the tables 1 to 4 . From the results it is clear that the Tanh transfer function along with LevenbergMarquardt learning rule shows optimum results.

Table 1: Mean Squared Error

\begin{tabular}{|l|l|l|l|l|l|}
\hline \multicolumn{5}{|l}{ Learning Rule } \\
\hline TF & A & B & C & D & E \\
\hline F & 0.0219 & 0.0239 & $\mathbf{0 . 0 0 5 6}$ & 0.0328 & 0.0769 \\
\hline G & 0.0888 & 0.0285 & 0.0083 & 0.1003 & 0.0231 \\
\hline H & 0.0358 & 0.0329 & 0.2 & 0.1053 & 0.0941 \\
\hline I & 0.0685 & 0.0494 & 0.0621 & 0.0887 & 0.0387 \\
\hline J & 0.098 & 0.0968 & 0.0368 & 0.0953 & 0.0941 \\
\hline K & 0.0959 & 0.0708 & 0.0695 & 0.0805 & 0.1112 \\
\hline
\end{tabular}

Table 2: Normalised Mean Squared Error

\begin{tabular}{|l|l|l|l|l|l|}
\hline TF & A & B & C & D & E \\
\hline F & 0.2023 & 0.2235 & $\mathbf{0 . 0 5 1 8}$ & 0.3046 & 0.7084 \\
\hline $\mathbf{G}$ & 0.8166 & 0.2656 & 0.0746 & 0.9217 & 0.2177 \\
\hline $\mathbf{H}$ & 0.3407 & 0.3078 & 2 & 0.9563 & 0.8672 \\
\hline $\mathbf{I}$ & 0.6311 & 0.4533 & 0.5696 & 0.8146 & 0.3612 \\
\hline $\mathbf{J}$ & 0.8991 & 0.8877 & 0.3412 & 0.8721 & 0.8602 \\
\hline $\mathbf{K}$ & 0.8815 & 0.6533 & 0.6396 & 0.7418 & 1.02 \\
\hline
\end{tabular}

Table 3: Correlation Coefficient

\begin{tabular}{|l|l|l|l|l|l|}
\hline TF & \multicolumn{1}{l|}{ A } & B & C & D & E \\
\hline F & 0.6587 & 0.8876 & $\mathbf{0 . 9 8 3 5}$ & 0.8375 & 0.5236 \\
\hline G & 0.434 & 0.8637 & 0.9686 & 0.3803 & 0.892 \\
\hline H & 0.7902 & 0.8443 & $-4 \mathrm{E}-17$ & 0.4202 & 0.3476 \\
\hline I & 0.6044 & 0.7395 & 0.6237 & 0.4197 & 0.8175 \\
\hline J & 0.3502 & 0.412 & 0.8228 & 0.4119 & 0.4107 \\
\hline K & 0.3176 & 0.5859 & 0.6003 & 0.4994 & -0.132 \\
\hline
\end{tabular}

Table 4: Specificity

\begin{tabular}{|l|r|l|l|l|l|}
\hline TF & \multicolumn{1}{|l|}{ A } & B & C & D & E \\
\hline F & 98.8 & 98.618 & 95.648 & 98.58 & 89.934 \\
\hline G & 90.742 & 96.022 & 95.515 & 87.47 & 96.287 \\
\hline
\end{tabular}




\begin{tabular}{|l|r|r|r|r|r|}
$\mathbf{H}$ & 93.897 & 96.402 & 79.841 & 90.05 & 88.03 \\
\hline $\mathbf{I}$ & 92.537 & 94.582 & 95.677 & 90.887 & 95.108 \\
\hline $\mathbf{J}$ & 87.365 & 87.625 & 94.384 & 89.138 & 88.306 \\
\hline $\mathbf{K}$ & 87.561 & 93.483 & 93.675 & 91.602 & 85.252 \\
\hline
\end{tabular}

Table 5: Sensitivity

\begin{tabular}{|l|l|l|l|l|l|}
\hline TF & A & B & C & D & E \\
\hline F & 91.914 & 90.753 & 98.868 & 90.162 & 50.594 \\
\hline G & 62.753 & 90.975 & 98.226 & 27.909 & 90.613 \\
\hline H & 77.723 & 89.539 & 45.33 & 58.919 & 37.59 \\
\hline I & 76.823 & 85.348 & 76.245 & 58.413 & 88.536 \\
\hline J & 30.754 & 41.283 & 82.507 & 41.663 & 39.225 \\
\hline K & 39.306 & 78.824 & 79.898 & 70.869 & 39.281 \\
\hline
\end{tabular}

Table 6: Precision

\begin{tabular}{|l|l|l|l|l|l|}
\hline TF & A & B & C & D & E \\
\hline F & 91.458 & 90.243 & 98.798 & 89.85 & 50.592 \\
\hline G & 54.851 & 90.307 & 98.073 & 24.996 & 89.609 \\
\hline H & 79.339 & 89.145 & 12.5 & 49.662 & 37.59 \\
\hline I & 70.827 & 83.625 & 77.224 & 56.988 & 87.595 \\
\hline J & 25.597 & 27.003 & 82.295 & 40.506 & 35.493 \\
\hline K & 25.455 & 75.553 & 78.146 & 62.427 & 12.219 \\
\hline
\end{tabular}

Table 7: Overall Accuracy

\begin{tabular}{|l|l|l|l|l|l|}
\hline TF & A & B & C & D & E \\
\hline F & 97.943 & 97.609 & 99.705 & 97.349 & 84.209 \\
\hline G & 86.469 & 97.319 & 99.518 & 78.155 & 97.093 \\
\hline $\mathbf{H}$ & 91.621 & 96.988 & 75.064 & 84.011 & 80.517 \\
\hline $\mathbf{I}$ & 91.172 & 95.09 & 93.156 & 86.554 & 96.343 \\
\hline $\mathbf{J}$ & 78.081 & 78.578 & 95.016 & 82.111 & 80.824 \\
\hline $\mathbf{K}$ & 78.601 & 92.468 & 93.302 & 88.737 & 74.985 \\
\hline
\end{tabular}

\section{CONCLUSION}

During this experimentation, multilayer perceptron neural network with Tanh transfer function and LevenbergMarquardt learning rule has given most optimum results for the performance parameters among all combination. The values of performance parameters are as follows:

Table 1 lists overall classification performance based on Mean Squared Error and is found to be 0.0056. Table 2 shows the normalized mean squared error it is 0.0518 . Table 3 contains correlation coefficient, which reflects amount of similarities among the signals, it is 0.98 . From table 4 specificity is 95.648 percent. Sensitivity is compared in table 5, it is 98.868 . The last parameter overall accuracy of classification is found to be 99.76 percent. In conclusion, the combination of network parameters used for the simulation has given the optimum results.

\section{REFERENCES}

[1] L. Biel, O. Petersson, L.P. PhilipsonWide,"ECG Analysis: a new approach in human identification," IEEE Trans. on Instrumentation and Measurement,Vol. 50(3), pp.808-812 (2001).

[2] A.K. Jain,A. Ross, S. Prabhakar, "An introduction to biometric recognition," IEEE Trans. Circuits. Syst. Video 14(0), 4-20 (2004).

[3] Fahim Sufi, Ibrahim Khalil, and JiankunHu, "ECG-Based Authentication," Handbook of information and Communication Security, Springer 2010.

[5] Y.Wang, F. Agrafioti, D. Hatzinakos, K. N. Plataniotis,"Analysis of human electrocardiogram for biometricrecognition," EURASIP Journal on Advances in Signal Process., Volume2008, 148658 (2008).Steven Bowbrick, Alex N. Borg, ECG Complete, Elsevier Limited, 2006.

[6] T.W. Shen,W.J. Tompkins, Y.H. Hu, "One-lead ECG for identity verification,"In Second Joint EMBS/BMES Conference Housten, TX USA (2002) pp. 62-63.

[7] S.A. Israel, J.M. Irvine, A. Cheng, M.D. Wiederhold, B.K. Wiederhold,"ECG to identify individuals,"Journal of Pattern Recognition Society. 38(2005), pp.133-142.

[8] Chan, Adrian D. C.; Hamdy, Mohyledin M.;Badre, Armin; Badee, Vesal, "Wavelet Distance Measure for Person Identification Using Electrocardiograms," IEEETransactions On Instrumentation And Measurement, Vol. 57, No. 2, pp. 3-16, February 2008.

[9] Yongbo Wan and JianchuYao,"A Neural Network to Identify Human Subjects with Electrocardiogram Signals," Proceedings of the World Congress on Engineering and Computer Science 2008, WCECS 2008.

[10] Yongjin Wang, FoteiniAgrafioti, DimitriosHatzinakos, Konstantinos N. Plataniotis, "Analysis of Electrocardiogram (ECG) for Biometric Recognition”, Journal on Advances in Signal Processing, Volume 2008, Article ID 148658.

[11] P. S. Gawande, S. A. Ladhake, "Investigation: Use of Electrocardiogram as a biometric over Longer Time Period", International Conference on Signal Processing and Communication (ICSC-2013), Dec 2013.

[12] Simon Haykin, "Neural Networks and Learning Machines", Third Edition, Pearson Education

[13] S. M. Jadhav, Dr. S. L. Nalbalwar, Dr. Ashok A. Ghatol, "Artificial Neural Network Based Cardiac Arrhythmia Classification Using ECG Signal Data", in Proc. Int. Conference on Electronics and Information Engineering ICEIE 2010, Japan, 2. 